\title{
Decreased Peak Drug Blood Concentration
}

National Cancer Institute

\section{Source}

National Cancer Institute. Decreased Peak Drug Blood Concentration. NCI Thesaurus. Code C54606.

Decreased maximum (peak) concentration of a drug observed after its administration. 